\title{
Short-Term Neonatal Outcomes in Diamniotic Twin Pregnancies Delivered after 32 Weeks and Indications of Late Preterm Deliveries
}

\author{
Alireza A. Shamshirsaz, MD ${ }^{1,2}$ Samadh F. Ravangard, DO ${ }^{2}$ Ali Ozhand, MD, PhD \\ Sina Haeri, MD, MHSA ${ }^{1}$ Amirhoushang A. Shamshirsaz, MD ${ }^{4}$ Naveed Hussain, MD ${ }^{5}$ Melissa Spiel, MD ${ }^{2}$ \\ Oluseyi Ogunleye, MD ${ }^{6}$ Rachel Billstrom, MD ${ }^{2}$ Alison Sadowski, MS ${ }^{2}$ Garry Turner, MD² \\ Diane Timms, DO, MS² James F. X. Egan, MD² Winston A. Campbell, MD²
}

${ }^{1}$ Department of Obstetrics and Gynecology, Baylor College of Medicine/Texas Children's Hospital, Houston, Texas

2 Department of Obstetrics and Gynecology, University of Connecticut Health Center, Farmington, Connecticut

${ }^{3}$ Department of Preventive Medicine, USC Keck School of Medicine, Los Angeles, California

${ }^{4}$ Department of Obstetrics and Gynecology, The George Washington University School of Medicine, Washington, District of Columbia

${ }^{5}$ Department of Pediatrics, University of Connecticut Health Center, Farmington, Connecticut

6 Department of Obstetrics and Gynecology, Waverly Women's Health Care, Columbia, South Carolina

Am J Perinatol 2014;31:365-372.
Address for correspondence Alireza A. Shamshirsaz, Pavilion for Women, Texas Children's Fetal Center, 6651 Main Street, Suite F1020, Houston, TX 77030 (e-mail: alirezashamshirsaz@yahoo.com; shamshir@bcm.edu).

\section{Abstract}

\section{Keywords}

- diamniotic twin

- neonatal outcomes

- late preterm births

- moderately preterm births
Objective We sought to compare neonatal outcomes in twin pregnancies following moderately preterm birth (MPTB), late preterm birth (LPTB), and term birth and determine the indications of LPTB.

Study Design We performed a retrospective cohort study. MPTB was defined as delivery between $32^{0 / 7}$ and $33^{6 / 7}$ weeks and LPTB between $34^{0 / 7}$ and $36^{6 / 7}$ weeks. The composite neonatal adverse respiratory outcome was defined as respiratory distress syndrome and/or bronchopulmonary dysplasia. The composite neonatal adverse nonrespiratory outcome included early onset culture-proven sepsis, necrotizing enterocolitis, retinopathy of prematurity, intraventricular hemorrhage, or periventricular leukomalacia. LPTB cases were categorized as spontaneous (noniatrogenic), evidence-based iatrogenic, and non-evidence-based (NEB) iatrogenic.

Results Of the 747 twin deliveries during the study period, 453 sets met the inclusion criteria with $22.7 \%(n=145)$ MPTB, $32.1 \%(n=206)$ LPTB, and $15.9 \%(n=102)$ term births. Compared with term neonates, the composite neonatal adverse respiratory outcome was increased following MPTB (relative risk [RR] 24; 95\% confidence interval [Cl] 3.0 to 193.6) and LPTB (RR 13.7; $95 \% \mathrm{Cl} 1.8$ to 101.8). Compared with term neonates, the composite neonatal adverse nonrespiratory outcome was increased following MPTB (RR 22.3; 95\% Cl 3.9 to 127.8) and LPTB (RR 5.5; 95\% Cl 1.1 to 27.6). Spontaneous delivery of LPTB was $63.6 \%(n=131 / 206)$ and the rate of iatrogenic delivery was $36.4 \%(n=75 / 206)$. The majority, $66.6 \%(n=50 / 75)$, of these iatrogenic received

December 25, 2012

accepted

January 2, 2013

published online

October 28, 2013
Copyright $\odot 2014$ by Thieme Medical Publishers, Inc., 333 Seventh Avenue, New York, NY 10001, USA. Tel: +1(212) 584-4662.
DOI http://dx.doi.org/ 10.1055/s-0033-1334458. ISSN 0735-1631. 
deliveries were deemed NEB, giving a total of $24.2 \%$ (50/206) NEB deliveries in LPTB group.

Conclusion Our data demonstrate a high rate of late preterm birth among twin pregnancies, with over half of nonspontaneous early deliveries due to NEB indications. Although our morbidity data will be helpful to providers in counseling patients, our finding of high NEB indications underscores the need for systematic evaluation of indications for delivery in LPTB twin deliveries. Furthermore, this may lead to more effective LPTB rate reduction efforts.

Late preterm births (LPTBs), which constitute more than $70 \%$ of all preterm deliveries ( $24^{0 / 7}$ to $36^{6 / 7}$ weeks' gestation), ${ }^{1}$ have recently garnered increased attention due to consistent demonstration of higher than previously expected neonatal morbidity and mortality in this group. In fact, this has led to the classification of preterm deliveries into very preterm birth (VPTB, $24^{0 / 7}$ to $31^{6 / 7}$ weeks), moderately preterm births (MPTB, $32^{0 / 7}$ to $33^{6 / 7}$ weeks), and LPTBs $\left(34^{\circ} / 7\right.$ to $36^{6 / 7}$ weeks). ${ }^{2-4}$ In an effort to decrease the prevalence of LPTB, there has been increased focus on the obstetric indications leading to these deliveries. Also, MPTB infants have significant prematurity-related morbidities of all preterm births in the United States. ${ }^{17}$

Unfortunately, these efforts have predominantly involved singleton pregnancies, and there remains a paucity of data regarding obstetric indications leading to LPTB in twin gestations. The morbidity associated with preterm births (PTBs) coupled with the increasing rates of twin gestations and their associated preterm deliveries make this an important public health concern. Consequently, the objective of this study was to compare neonatal outcomes in twin pregnancies following MPTB, LPTB, and term birth in diamniotic twin pregnancies and to identify obstetric indications leading to early nonspontaneous delivery.

\section{Methods and Materials}

We conducted a retrospective cohort analysis of all twin deliveries at our institution between January 1, 1991, and January 1, 2011. Approval from the Committee for the Protection of Human Subjects Institutional Review Board at the University of Connecticut Health Center was obtained prior to data collection.

Inclusion criteria were twin gestation with two live-born infants and delivery after $32^{0 / 7}$ weeks of gestation. Exclusion criteria included cases complicated by pregnancies with major congenital malformations, Rh alloimmunization, monoamniocity, and twin-to-twin transfusion syndrome. Subjects were categorized as MPTB (delivery between $32^{0 / 7}$ and $33^{6 / 7}$ weeks), LPTB (delivery between $34^{0 / 7}$ and $36^{6 / 7}$ weeks), and term birth (TB, delivery after $37^{0 / 7}$ ). The TB group served as the referent population.

Demographic data were abstracted from the prenatal and inpatient records. Gestational age, chorionicity, and amnionicity were determined from prenatal records. In cases of uncertain last menstrual period, ultrasound-determined gestational age was used. Obstetric variables of interest included gestational hypertension and preeclampsia, gestational and pregestational diabetes, and treatment with antenatal corticosteroids and/or magnesium sulfate were abstracted from the prenatal and inpatient records.

Neonatal outcomes of interest were defined as respiratory distress syndrome based on the clinical findings of respiratory distress and chest $\mathrm{X}$-ray findings of a diffuse reticulogranular pattern with air bronchograms ${ }^{5}$; days of mechanical ventilation and/or continuous or intermittent positive airway pressure; intraventricular hemorrhage based on the Papile classification of cranial ultrasound findings of blood in the germinal matrix or ventricular system with or without ventricular dilatation ${ }^{6}$; necrotizing enterocolitis based on Bell's classification (stage II and above) ${ }^{7}$; periventricular leukomalacia as diagnosed by cerebral ultrasound findings of increased echogenicity and cystic lesions in the periventricular white matter ${ }^{8}$; retinopathy of prematurity (ROP) based on pediatric ophthalmologic exam using the international classification of $\mathrm{ROP}^{9}$; bronchopulmonary dysplasia diagnosed by the presence of chronic respiratory distress with an oxygen requirement beyond 28 days of life, accompanied by characteristic chest roentgenogram findings ${ }^{10}$; early culture-proven sepsis defined as positive bacterial culture from samples obtained within the first 3 days of life; and length of hospitalization based on final discharge to a nonmedical facility. The composite neonatal adverse respiratory outcome was defined as respiratory distress syndrome and/or bronchopulmonary dysplasia. The composite neonatal adverse nonrespiratory outcome included early onset culture-proven sepsis, necrotizing enterocolitis, ROP, intraventricular hemorrhage, or periventricular leukomalacia.

We categorized the indication for LPTB using current recommendations endorsed by the American Congress of Obstetricians and Gynecologists (ACOG) $)^{11}$ or published expert opinion (level III evidence). Spontaneous (noniatrogenic) causes of preterm delivery included cases of either premature rupture of membranes or preterm labor with intact membranes. Iatrogenic deliveries (indicated) were further categorizes as follows: deliveries based on obstetric indications supported by ACOG guidelines and /or expert opinion (level III evidence and expert opinion) were defined as evidencebased (EB), and deliveries based on obstetric indications not 
Table 1 Categorization of indications for iatrogenic delivery

\begin{tabular}{|l|l|}
\hline Evidence-based & Non-evidence-based \\
\hline Severe preeclampsia/eclampsia/HELLP syndrome & Mild preeclampsia/gestational hypertension \\
\hline Nonreassuring fetal status & IUGR with normal testing and adequate interval growth \\
\hline Acute abruption & Oligohydramnios \\
\hline & Prior classical cesarean delivery \\
\hline & Placenta previa \\
\hline & Cholestasis of pregnancy \\
\hline & No clear indication (elective) \\
\hline
\end{tabular}

Abbreviations: HELLP syndrome, hemolysis, elevated liver enzymes, low platelet count; IUGR, intrauterine growth restriction.

supported by either of these were labeled non-EB (NEB; - Table 1). All the NEB deliveries with no clear reason defined as elective.

Each twin was treated as one observation in the analysis. We created three categories of delivery time and compared other variables across these categories. We used analysis of variance to compare parametric continuous variables and Kruskal-Wallis test to compare nonparametric continuous variables across categories of delivery time. To compare the distribution of categorical variables between groups of delivery time, chi-square test or Fisher exact test was used. Risk of having a respiratory or nonrespiratory outcome, was assessed in MPTB and LPTB neonates relative to term neonates, after adjustment for antenatal corticosteroid and magnesium sulfate treatment, using multiple logistic regression models. We additionally adjusted our models for maternal age, diabetes, chronic hypertension, prior preterm birth, and in vitro fertilization. In this study, the unit of analysis is a twin. To account for a potential nonindependence of twin outcomes, we also used generalized estimating equation models. A significance level of 0.05 was used to reject the null hypothesis. Data was analyzed using SAS version 9.2.

\section{Results}

Of the 747 twin deliveries during the study period, 453 sets met the inclusion criteria with $22.7 \%(n=145)$ MPTB, $32.1 \%$ $(n=206)$ LPTB, and $15.9 \%(n=102)$ term births (-Fig. 1$)$.

Maternal factors were similar among the three groups except for older age among women who delivered at term (-Table 2). There were no neonatal deaths in the three groups. The rates of the adverse neonatal outcomes as defined by composite outcomes (respiratory and nonrespiratory) were significantly higher in those twins with MPTB and LPTB compared with term births (-Table 3 ). The relative risk of neonatal adverse outcomes (composite respiratory and nonrespiratory outcomes) increased progressively from MPTB to LPTB to TB with and without adjustment for other confounding factors ( - Tables $\mathbf{4 A}$ and $\mathbf{4 B}$ ). Neonatal characteristics and the specific short-term neonatal outcomes of their 906 infants are shown in -Table 5.

Obstetric indications such as preeclampsia or gestational hypertension, and antepartum interventions such as antena- tal corticosteroid or magnesium sulfate therapy were significantly higher in earlier gestations (-Table 6).

Indications for delivery in the LPTB were shown in -Table 7. Spontaneous delivery of LPTB was $63.6 \%$ ( $n=131 / 206)$ and the rate of iatrogenic delivery in this late preterm cohort was $36.4 \%(n=75 / 206)$. The majority, $66.6 \%(n=50 / 75)$, of these iatrogenic deliveries were deemed NEB, giving a total of $24.2 \%$ (50/206) NEB deliveries in LPTB group. All the elective deliveries were between $36^{0 / 7}$ and $36^{6 / 7}$ weeks. The mean gestational age at delivery was higher in the NEB group (36.1 weeks) than in the EB group (35.2 weeks; $p<0.001$ ). In the univariate analysis, neonates in the NEB group had a lower median length of stay in the neonatal intensive care unit (14 days versus 5 days, $p<0.032$ ). The rates of the adverse neonatal composite outcomes (respiratory and nonrespiratory) were not significantly different between NEB and EB groups.

\section{Discussion}

In this cohort study, we sought out to estimate the increased perinatal risk associated with MPTB and LPTB in uncomplicated diamniotic twin pregnancies and to identify obstetric



Fig. 1 Proportion of twin pairs delivering moderately preterm, late preterm, and term births in the study. 
Table 2 Maternal characteristics

\begin{tabular}{|c|c|c|c|c|}
\hline & $\begin{array}{l}\text { MPTB } \\
\text { (145 twin pairs) }\end{array}$ & $\begin{array}{l}\text { LPTB } \\
\text { (206 twin pairs) }\end{array}$ & $\begin{array}{l}\text { TB } \\
\text { (102 twin pairs) }\end{array}$ & $p$ value \\
\hline Maternal age, y (SD) & $29.6(5.8)$ & $31(6.1)$ & $32.8(6)$ & $<0.0001$ \\
\hline Mean gestational age at delivery, wk (SD) & $33(0.6)$ & $35.3(0.9)$ & $37.8(0.6)$ & $<0.0001$ \\
\hline Prior preterm births (\%) & $11(7.6)$ & $23(11.2)$ & $5(4.9)$ & 0.0331 \\
\hline Prior term births (\%) & $65(44.8)$ & $82(39.8)$ & $46(45.1)$ & 0.2975 \\
\hline Start BMI (SD) & $25.9(6.2)$ & $26.2(6.6)$ & $25(5.7)$ & 0.1622 \\
\hline Smoking (\%) & $13(9.0)$ & $11(5.3)$ & $7(6.9)$ & 0.1729 \\
\hline Race (\%) & & & & 0.3457 \\
\hline Caucasian & $115(79.3)$ & $167(81.1)$ & $72(70.6)$ & \\
\hline African-American & $3(2.1)$ & $5(2.4)$ & $7(6.9)$ & \\
\hline Hispanic & $13(9.0)$ & $14(6.8)$ & $10(9.8)$ & \\
\hline Other & $14(9.6)$ & $20(9.7)$ & $13(12.7)$ & 0.2834 \\
\hline Diabetes (\%) & $13(9.0)$ & $25(12.1)$ & $6(5.9)$ & 0.0529 \\
\hline CHTN (\%) & $19(13.1)$ & $37(18.0)$ & $12(11.8)$ & 0.0524 \\
\hline IVF (\%) & $9(8.8)$ & $32(15.5)$ & $17(11.7)$ & 0.0343 \\
\hline Mono-Di twins (\%) & $30(20.7)$ & $35(17.0)$ & $16(15.7)$ & 0.2938 \\
\hline
\end{tabular}

Abbreviations: BMI, body mass index; CHTN, chronic hypertension; IVF, in vitro fertilization; LPTB, late preterm birth; Mono-Di twins, monochorionicdiamniotic twins; MPTB, moderately preterm birth; TB, term birth; SD, standard deviation.

Note: Results are mean \pm standard deviation or $n(\%)$.

Table 3 The composite neonatal adverse outcomes

\begin{tabular}{|l|l|l|l|l|}
\hline & $\begin{array}{l}\text { MPTB } \\
(290 \text { neonates) }\end{array}$ & $\begin{array}{l}\text { LPTB } \\
(412 \text { neonates })\end{array}$ & $\begin{array}{l}\text { TB } \\
(204 \text { neonates) }\end{array}$ & $p$ value \\
\hline Respiratory outcome (\%) & $50(17.2)$ & $35(8.5)$ & $1(0.5)$ & $<0.0001$ \\
\hline Nonrespiratory outcomes (\%) & $29(10.0)$ & $16(3.9)$ & $2(1)$ & $<0.0001$ \\
\hline
\end{tabular}

Abbreviations: LPTB, late preterm birth; MPTB, moderately preterm birth; TB, term birth.

Note: Results are $n(\%)$. Primary respiratory outcomes: respiratory distress syndrome and bronchopulmonary dysplasia. Primary nonrespiratory outcomes: early onset culture-proven sepsis, necrotizing enterocolitis, retinopathy of prematurity, intraventricular hemorrhage, periventricular leukomalacia.

Table 4A Relative risk of adverse neonatal outcomes among LPTB and MPTB twin gestations compared with TB twin gestations

\begin{tabular}{|l|l|l|l|l|}
\hline Composite neonatal outcomes & Delivery time & \multicolumn{4}{|c|}{ OR (95\% Wald confidence limits) } \\
\hline & & Crude & Adjusted $^{\text {a }}$ & Adjusted $^{\text {b }}$ \\
\hline Respiratory & LPTB vs. TB & $18.8(2.6-138.6)$ & $17.6(2.4-129.7)$ & $16.7(2.2-123.4)$ \\
\hline \multicolumn{7}{|l|}{ MPTB vs. TB } & $42.3(5.8-308.8)$ & $35.2(4.7-261.9)$ & $29.9(4-223.9)$ \\
\hline & LPTB vs. TB & $4.1(0.9-17.9)$ & $4.7(1.1-20.6)$ & $4.3(1-19)$ \\
\hline Nonrespiratory & MPTB vs. TB & $11.2(2.6-47.5)$ & $17.6(4.1-76.5)$ & $18.4(4.2-81.3)$ \\
\hline
\end{tabular}

Abbreviations: LPTB, late preterm birth; MPTB, moderate preterm birth; TB, term birth.

Note: Primary respiratory outcomes: respiratory distress syndrome, and bronchopulmonary dysplasia. Primary nonrespiratory outcomes: early onset culture-proven sepsis, NEC, necrotizing enterocolitis; ROP, retinopathy of prematurity; intraventricular hemorrhage, periventricular leukomalacia.

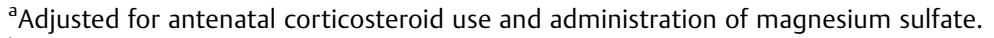

${ }^{\mathrm{b}}$ Additionally adjusted for maternal age, diabetes, chronic hypertension, prior preterm birth, and in vitro fertilization. 
Table 4B Relative risk of adverse neonatal outcomes among late and moderate preterm birth Twin gestations compared with term twin gestations (calculated using generalized estimating equation models to account for a nonindependence of twin outcomes)

\begin{tabular}{|c|c|c|c|c|}
\hline \multirow[t]{2}{*}{ Composite neonatal outcomes } & \multirow[t]{2}{*}{ Delivery time } & \multicolumn{3}{|c|}{ OR (95\% Wald confidence limits) } \\
\hline & & Crude & Adjusted $^{\mathrm{a}}$ & Adjusted $^{\mathrm{b}}$ \\
\hline \multicolumn{5}{|l|}{ Respiratory } \\
\hline & LPTB vs. TB & $18.3(2.6-128.6)$ & $14.9(2.1-108.4)$ & $13.7(1.8-101.8)$ \\
\hline & MPTB vs. TB & 41 (5.9-286.5) & $29.3(3.7-232.3)$ & $24(3-193.6)$ \\
\hline \multicolumn{5}{|l|}{ Nonrespiratory } \\
\hline & LPTB vs. TB & $4(0.9-17.6)$ & $5.6(1.2-27.4)$ & $5.5(1.1-27.6)$ \\
\hline & MPTB vs. TB & $11(2.6-46.8)$ & $20.8(3.9-110.3)$ & $22.3(3.9-127.8)$ \\
\hline
\end{tabular}

Abbreviations: LPTB, late preterm birth; MPTB, moderate preterm birth; TB, term birth.

Note: Primary respiratory outcomes: respiratory distress syndrome, and bronchopulmonary dysplasia. Primary nonrespiratory outcomes: early onset culture-proven sepsis, NEC, necrotizing enterocolitis; ROP, retinopathy of prematurity; intraventricular hemorrhage, periventricular leukomalacia.

${ }^{a}$ Adjusted for antenatal corticosteroid use and administration of magnesium sulfate.

${ }^{b}$ Additionally adjusted for maternal age, diabetes, chronic hypertension, prior preterm birth, and in vitro fertilization.

indications leading to early nonspontaneous delivery. We found that the majority of nonspontaneous LPTBs were NEB. In fact, $24.2 \%$ of our late preterm cohort was delivered for NEB indications, many of which could be classified as perhaps "avoidable." Due to the well-established morbidity associated with LPTB, coupled with the higher than anticipated rate of NEB deliveries, our findings underscore the need for a thorough examination of indications leading to LPTB in twin pregnancies. Indeed, our results implicated mild preeclampsia/gestational hypertension (10.2\%), intrauterine growth restriction with reassuring antenatal testing and/or oligohydramnios (6.3\%), and no clear cause (elective; $5.8 \%$ ) as leading causes for NEB LPTB.

The rate of multifetal pregnancies, specifically twin gestations, has risen in recent years, most likely as a consequence of fertility treatments and delayed childbearing. ${ }^{12,13}$

Table 5 Neonatal characteristics and individual outcomes

\begin{tabular}{|l|l|l|l|l|}
\hline & $\begin{array}{l}\text { MPTB } \\
(290 \text { neonates })\end{array}$ & $\begin{array}{l}\text { LPTB } \\
(412 \text { neonates })\end{array}$ & $\begin{array}{l}\text { TB } \\
(204 \text { neonates })\end{array}$ & $p$ value \\
\hline Birth weight, g (SD) & $1,894.3(327.6)$ & $2,329.1(430.2)$ & $2,749.6(380.7)$ & $<000.1$ \\
\hline Female gender (\%) & $140(48.3)$ & $228(55.3)$ & $112(54.9)$ & 0.1314 \\
\hline RDS, $n$ (\%) & $50(17.2)$ & $33(8.0)$ & $1(0.5)$ & $<0.0001$ \\
\hline Early sepsis, $n$ (\%) & $22(7.5)$ & $13(3.1)$ & $1(0.5)$ & 0.0001 \\
\hline NEC, $n$ (\%) & $2(0.6)$ & $3(0.7)$ & $0(0)$ & 0.6190 \\
\hline BPD, $n$ (\%) & $7(2.4)$ & $5(1.2)$ & $0(0)$ & 0.0545 \\
\hline ROP, $n$ (\%) & $3(1.0)$ & $0(0)$ & $0(0)$ & 0.0439 \\
\hline IVH, $n$ (\%) & $3(1.0)$ & $0(0)$ & $0(0)$ & $1(0.5)$ \\
\hline I and II & $1(0.3)$ & $0(0)$ & $1(0.5)$ & 0.0303 \\
\hline III and IV & $8(2.7)$ & $7(1.7)$ & $2(1.0)$ & 0.1764 \\
\hline Apgar 5 min $<7, n$ (\%) & $55(18.9)$ & $31(7.5)$ & $5(2.4)$ & $<0.0001$ \\
\hline MV, $n$ (\%) & $109(37.6)$ & $81(19.6)$ & $17(8.3)$ & $<0.0001$ \\
\hline CPAP, $n$ (\%) & $290(100.0)$ & $262(63.6)$ & $5(2-113)$ & $<0.0001$ \\
\hline NICU admission, $n$ (\%) & $20(4-101)$ & $13(1-56)$ & $0(0)$ & NA \\
\hline Median length of NICU, d (range) & $0(0)$ & $0(0)$ & 0001 \\
\hline PVL &
\end{tabular}

Abbreviations: BPD, bronchopulmonary dysplasia; CPAP, continuous or intermittent positive airway pressure; IVH, intraventricular hemorrhage; LPTB, late preterm birth; MPTB, moderate preterm birth; MV, mechanical ventilation; NA, not applicable; NEC, necrotizing enterocolitis; NICU, neonatal intensive care unit; PVL, periventricular leukomalacia; RDS, respiratory distress syndrome; ROP, retinopathy of prematurity; SD, standard deviation; TB, term birth. 
Table 6 Clinical characteristics

\begin{tabular}{|l|l|l|l|l|}
\hline & $\begin{array}{l}\text { MPTB } \\
(145 \text { mothers })\end{array}$ & $\begin{array}{l}\text { LPTB } \\
(206 \text { mothers })\end{array}$ & $\begin{array}{l}\text { TB } \\
(102 \text { mothers })\end{array}$ & $p$ value \\
\hline ACS & $86(59.3)$ & $75(36.4)$ & $18(17.6)$ & $<0.0001$ \\
\hline Mag sulfate & $79(54.4)$ & $62(30.1)$ & $12(11.7)$ & $<0.0001$ \\
\hline Preeclampsia or PIH & $15(10.3)$ & $17(8.2)$ & $4(3.9)$ & $<0.0001$ \\
\hline PPROM & $48(33.1)$ & $40(19.4)$ & 0 & $<0.0001$ \\
\hline Cesarean delivery & $94(64.8)$ & $142(68.9)$ & $70(68.6)$ & 0.4533 \\
\hline V/C & $11(7.6)$ & $10(4.8)$ & $2(1.9)$ & 0.0008 \\
\hline
\end{tabular}

Abbreviations: ACS, antenatal corticosteroid; LPTB, late preterm birth; Mag, administration of magnesium; MPTB, moderate preterm birth; PIH, pregnancy-induced hypertension; PPROM, preterm premature rupture of membranes; TB, term birth; $V / C$, first twin vaginal delivery and second cesarean delivery.

Table 7 Indications for LPTB in twin pregnancies $(n=206)$

\begin{tabular}{|l|l|l|}
\hline Indications & $\boldsymbol{n}(\%)$ & Cause of LPTB \\
\hline Spontaneous preterm labor & $69(33.6)$ & Spontaneous \\
\hline PPROM & $41(19.9)$ & Spontaneous \\
\hline Spontaneous preterm labor/PPROM & $21(10.2)$ & Spontaneous \\
\hline Severe preeclampsia/eclampsia/HELLP syndrome & $15(7.2)$ & latrogenic EB \\
\hline Nonreassuring fetal status & $9(4.4)$ & latrogenic EB \\
\hline Mild preeclampsia/gestational hypertension & $21(10.2)$ & latrogenic NEB \\
\hline Intrauterine growth restriction and/or oligohydramnios & $13(6.3)$ & latrogenic NEB \\
\hline No clear indication (elective) & $12(5.8)$ & latrogenic NEB \\
\hline Other indications & $5(2.4)$ & latrogenic EB and NEB \\
\hline
\end{tabular}

Abbreviations: EB, evidence-based; HELLP syndrome; hemolysis, elevated liver enzymes, low platelet count; LPTB, late preterm birth; NEB, nonevidence-based; PPROM, preterm premature rupture of membranes.

${ }^{a}$ One abruption, one cholestasis of pregnancy, two placentae previae, one prior classical cesarean.

Similarly, between 1980 and 2006, the percentage of births that were preterm rose from 9.4 to $12.7 \%$-a rise of nearly $30 \%{ }^{1,13,14}$ The percentage of twins delivered preterm has risen more than singletons, from 48 to $60 \%$ in the last two decades. ${ }^{14}$ Consequently, there has been increased focus on strategies aimed at decreasing the rate of LPTBs, with attention on the specific indications for delivery in these cases. Similar to findings by Gyamfi-Bannerman and colleagues, the majority of nonspontaneous LPTB in our cohort were NEB. ${ }^{15}$ The $24.2 \%$ rate of NEB deliveries in our LPTB patients is alarming. Even EB indications for LPTB are still only based on level III evidence/expert opinion. ${ }^{11}$ This finding underscores the need for critical examination of all the indications for delivery in these patients.

The concept of composite adverse respiratory and nonrespiratory outcomes has previously been well utilized. ${ }^{16} \mathrm{We}$ demonstrated a progressive increase in composite neonatal morbidity in twin pregnancies that ended with MPTB and LPTB compared with term deliveries. These findings are similar to and support those of the twin outcome study from the Eunice Kennedy Shriver National Institute of Child Health and Human Development Maternal-Fetal Medicine
Units Network. ${ }^{16}$ We found respiratory distress syndrome to be the most common individual morbidity with a 17 -fold increase among MPTB and an 8-fold increase in LPTB compared with term twins.

Infants born moderately preterm constitute approximately $32 \%$ of all preterm births in the United States, with significant prematurity-related morbidities. ${ }^{17}$ In one study, $45 \%$ of these babies required assisted ventilation and had a readmission rate within 3 months of $11.2 \%{ }^{17}$ Similarly, we found significantly higher composite respiratory and nonrespiratory morbidity in MPTB when compared with LPTB and TB neonates. Thus a significant portion of neonatal intensive care unit costs can be attributed to this group of patients, further highlighting the need to reevaluate the risks, benefits, and cost implications of earlier delivery in this group of patients.

We also need to weigh the risk of adverse neonatal outcomes against the risk of stillbirth that increases with gestation. Perinatal mortality among twin births declined close to $40 \%$ between 1989 and $2000 .^{18}$ This temporal trend of increasing twin preterm birth may actually be beneficial insofar as preventing perinatal death and may be a topic worthy of continued research. ${ }^{19}$ 
This study has some limitations that merit discussion. This study was retrospective in design and relied on chart review to establish diagnoses. Not all the pregnancies were dated by early ultrasound examination and as a result, the percentage of LTPBs is likely overestimated. It is possible that some diagnoses were over- or underdiagnosed, which has the potential to bias our results. Although we did not consider NEB deliveries as indicated, they may have been. However, to the best of our ability, we could not see an evidence-based reason barring expectant management. Nevertheless, this is one of the largest studies in twin pregnancies examining the indications and outcomes for LPTB.

\section{Conclusions}

Our data demonstrate a high rate of LPTB among twin pregnancies, with over half of nonspontaneous early deliveries due to NEB indications. Although our morbidity data will be helpful to providers in counseling patients, our finding of high NEB indications underscores the need for systematic evaluation of indications for delivery in LPTB twin deliveries. Furthermore, this may lead to more effective LPTB rate reduction efforts.

\section{Acknowledgment}

This article is dedicated to the memory of James F. X. Egan, our dear friend, colleague, and mentor.

\section{References}

1 Davidoff MJ, Dias T, Damus K, et al. Changes in the gestational age distribution among U.S. singleton births: impact on rates of late preterm birth, 1992 to 2002. Semin Perinatol 2006;30:8-15

2 Martin JA, Hamilton BE, Sutton PD, Ventura SJ, Mathews TJ, Osterman MJ. Births: final data for 2008. Natl Vital Stat Rep 2010;59:1, 3-71

3 Raju TN, Higgins RD, Stark AR, Leveno KJ. Optimizing care and outcome for late-preterm (near-term) infants: a summary of the workshop sponsored by the National Institute of Child Health and Human Development. Pediatrics 2006;118:1207-1214

4 Committee on Obstetric Practice. ACOG committee opinion No. 404 April 2008. Late-preterm infants. Obstet Gynecol 2008;111: 1029-1032
5 Boyle RJ, Oh W. Respiratory distress syndrome. Clin Perinatol 1978;5:283-297

6 Papile LA, Burstein J, Burstein R, Koffler H. Incidence and evolution of subependymal and intraventricular hemorrhage: a study of infants with birth weights less than 1,500 gm. J Pediatr 1978;92: 529-534

7 Banker BQ Larroche J-C. Periventricular leukomalacia of infancy. A form of neonatal anoxic encephalopathy. Arch Neurol 1962;7: 386-410

8 Volpe JJ. Hypoxic-ischemic encephalopathy: neuropathology and pathogenesis. In: Neurology of the Newborn. 3rd ed. Philadelphia, PA: W.B. Saunders; 1987

9 Anonymous; The Committee for the Classification of Retinopathy of Prematurity. An international classification of retinopathy of prematurity. Arch Ophthalmol 1984;102:1130-1134

10 Northway WH Jr, Rosan RC, Porter DY. Pulmonary disease following respirator therapy of hyaline-membrane disease. Bronchopulmonary dysplasia. N Engl J Med 1967;276:357-368

11 Spong CY, Mercer BM, D'alton M, Kilpatrick S, Blackwell S, Saade G. Timing of indicated late-preterm and early-term birth. Obstet Gynecol 2011;118(2 Pt 1):323-333

12 Reynolds MA, Schieve LA, Martin JA, Jeng G, Macaluso M. Trends in multiple births conceived using assisted reproductive technology, United States, 1997-2000. Pediatrics 2003;111(5 Pt 2): 1159-1162

13 Martin JA, Hamilton BE, Sutton PD, et al; Centers for Disease Control and Prevention National Center for Health Statistics National Vital Statistics System. Births: final data for 2005. Natl Vital Stat Rep 2007;56:1-103

14 Martin JA, Kung HC, Mathews TJ, et al. Annual summary of vital statistics: 2006. Pediatrics 2008;121:788-801

15 Gyamfi-Bannerman C, Fuchs KM, Young OM, Hoffman MK. Nonspontaneous late preterm birth: etiology and outcomes. Am J Obstet Gynecol 2011;205:e1-e6

16 Refuerzo JS, Momirova V, Peaceman AM, et al; Eunice Kennedy Shriver National Institute of Child Health and Human Development Maternal-Fetal Medicine Units Network. Neonatal outcomes in twin pregnancies delivered moderately preterm, late preterm, and term. Am J Perinatol 2010;27:537-542

17 Escobar GJ, McCormick MC, Zupancic JA, et al. Unstudied infants: outcomes of moderately premature infants in the neonatal intensive care unit. Arch Dis Child Fetal Neonatal Ed 2006;91: F238-F244

18 Ananth CV, Joseph KS, Demissie K, Vintzileos AM. Trends in twin preterm birth subtypes in the United States, 1989 through 2000: impact on perinatal mortality. Am J Obstet Gynecol 2005;193(3 Pt 2):1076-1082

19 Ananth CV, Vintzileos AM. Epidemiology of preterm birth and its clinical subtypes. J Matern Fetal Neonatal Med 2006;19: 773-782 